\title{
Validity of Immunochromatographic Test for Antibody in Diagnosis of Typhoid Fever in Children Admitted in A Tertiary Care Hospital
}

\author{
Zabeen Choudhury ${ }^{1 *}$ \\ Mohammed Rezaul Karim² \\ Rasheda Samad ${ }^{1}$ \\ Shanjana Islam ${ }^{3}$ \\ 'Department of Pediatrics \\ Chittagong Medical College Hospital \\ Chittagong, Bangladesh. \\ ${ }^{2}$ Department of Pediatrics \\ Cox's Bazar Medical College \\ Cox's Bazar, Bangladesh. \\ ${ }^{3}$ Department of Paediatrics \\ Bangabandhu Memorial Hospital (USTC) \\ Chittagong, Bangladesh.
}

\begin{abstract}
Background: To determine the Validity of Immunochromatographic Test (ICT) in diagnosis of typhoid fever in children admitted in a tertiary care hospital. Methods: This cross sectional study was carried out the in Pediatric \& Medicine wards of Chittagong Medical College Hospital $(\mathrm{CMCH})$, Chittagong during the period July 2012 to June 2013. A total number of 150 clinically suspected cases of typhoid fever (Age $>6$ months to18 years) were enrolled in this study. After taking informed written consent, detailed history \& clinical examination were completed. A blood culture sample was taken on the day of admission before starting antibiotic. On the 5th day onwards of appearance of fever, blood sample was taken to perform ICT. Patients received standard medical treatment of the admitting wards. Results: Blood $\mathrm{C} / \mathrm{S}$ for Salmonella typhi was found positive in $16(10.7 \%)$ cases. Positive ICT for typhoid fever was found in $37(24.7 \%)$ cases. Among then, IgM was $18(12.0 \%)$ $\operatorname{IgM}+\operatorname{IgG}$ were $8(5.3 \%)$ and $\operatorname{IgG}$ was $11(7.3 \%)$. ICT found true positive in 14 , false positive in 23, false negative in 2 and true negative in 111 cases, where blood culture considered as gold standard. The difference was statistically significant $(\mathrm{p}<0.05)$ between two groups. Immunochromatographic Test (ICT) showed sensitivity $87.5 \%$, specificity $82.8 \%$, accuracy $83.3 \%$, positive predictive value $37.8 \%$ and negative predictive value $98.2 \%$ for identification of typhoid fever. Conclusion: The present study has shown high sensitivity \& specificity of ICT, it can be used as a useful \& prospectful diagnostic tool.
\end{abstract}

Key words: Typhoid fever; Immunochromatographic Test (ICT); Blood culture.

\section{INTRODUCTION}

Typhoid fever is an acute systemic infection caused by Salmonella enterica serotype Typhi or Paratyphi. This disease is endemic in most developing countries, including South and Southeast Asia (Including Indonesia) Central America and other countries which are populous, have high urbanization and a lack of proper hygiene and sanitation (Parry et al. 2002) ${ }^{1}$. The worldwide incidence of typhoid fever is estimated to be approximately 16 million cases annually, of which 7 million cases occur in Southeast Asia. More than 600,000 people die due to this disease each year (Ivanoff, $1995)^{2}$.

The isolation of S. Typhi or S. Paratyphi A from blood, bone marrow, rose spots or other sterile sites provides the most conelusive confirmation of typhoid fever. Therefore, culture should be considered as the gold standard and used for evaluating all diagnostic tests, irrespective of their level of sophistication (WHO 2003) ${ }^{3}$. Bacterial isolation confirms the clinical diagnosis and allows antimicrobialsusceptibility testing which can direct appropriate therapy yet it is only positive in approximately $40-60 \%$ of presumptive cases (Gilman et al. 1975 and GuerraCaceres et al.2011 $)^{4,5}$.

\section{Dr. Zabeen Choudhury}

Resident Physician

Department of Pediatrics

Chittagong Medical College Hospital

Chittagong, Bangladesh.

Mobile : +88 01711747415

Email: zabeen.chowdhury9@gmail.com 
ICDDR, B in 2001 conducted a study at Kamalapur in Dhaka and concluded approximate incidence of typhoid fever in our population documented by positive blood culture is 3.9 episodes per year per 1000 populations.

The S. Typhi-specific antibodies from the suspected patients are detected by their ability to inhibit the binding between colored indicator particles that are coated with a monoclonal antibody $(\mathrm{mAb})$ specific for the S. Typhi O9 LPS antigen, and magnetic particles that are coated with S. Typhi LPS. Typhidot (Malaysian Biodiagnostic Research SDN BHD, Kuala Lumpur, Malaysia), was developed for the detection of specific IgM and IgG against a $50-\mathrm{kD}$ S. Typhi outer membrane protein (Choo et al. 1994 and Jackson et al.1995) $)^{6,7}$. Typhidot is an ELISA-based method, miniaturized into an immunodot test format.

Narayanappa et al. (2010) compared the sensitivity and specificity of Typhidot-M and Widal test with blood culture (Gold standard) for diagnosing typhoid fever in 105 children aged 1-15 years admitted with clinical suspicion of typhoid fever $^{8}$. Of the 105 cases, blood culture was positive for S.typhi in $41(39 \%)$ children, widal test was positive in $48(45.7 \%)$ and Typhidot-M was positive in $78(74.3 \%)$ cases. Sensitivity and specificity of Typhidot-M was $92.6 \%$ and $37.5 \%$ while sensitivity and specificity of Widal test was $34.1 \%$ and $42.8 \%$, respectively. In children with fever of less than 7 days duration, Typhidot-M was positive in $97 \%$, compared to $24.2 \%$ by widal test. Typhidot-M is a simple and sensitive test for early diagnosis of typhoid fever in children.

Pastoora et al. (2008) developed a point-of-care test for the serodiagnosis of typhoid fever in the format of an immunochromatographic lateral flow assay ${ }^{9}$. The sensitivity of this typhoid fever IgM flow assay for samples collected at $1 \mathrm{st}$ diagnosis from patients with culture-confirmed typhoid fever was determined to be $59.3 \%$. The sensitivity ranged from $41.2 \%$ to $89.5 \%$, depending on the duration of illness. A specificity of $97.8 \%$ was calculated based on results obtained for patients with clinical suspicion of typhoid fever that was later excluded.

Tubex ${ }^{\mathrm{TM}}$ and Typhidot ${ }^{\circledR}$, rapid tests for typhoid fever, performed well in evaluations conducted in hospital settings among patients with culture-confirmed typhoid fever. Naheed et al. (2008) evaluated these tests in a community clinic in Bangladesh. Blood samples were obtained from 867 febrile patients for culture, Typhidot ${ }^{\circledR}$ and Tubex ${ }^{\mathrm{TM}}$ tests. Considering the 43 blood culture-confirmed cases of typhoid fever as typhoid positive and the 24 other confirmed bacteraemia cases as typhoid negative, Tubex ${ }^{\mathrm{TM}}$ was $60 \%$ sensitive and $58 \%$ specific, with $90 \%$ positive and $58 \%$ Negative Predictive Values (NPVs) Typhidot ${ }^{\circledR}$ was $67 \%$ sensitive and $54 \%$ specific, with $85 \%$ positive and $81 \%$ NPVs. When blood culture-negative patients and other bacteraemia cases together were considered typhoid negative, positive predictive values were only $14 \%$ for Tubex ${ }^{\mathrm{TM}}$ and $13 \%$ for Typhidot ${ }^{\circledR}$ increasing to only $38 \%$ and $20 \%$ when restricted to patients with 7 days of fever. The investigators conclude that the value of Tubex ${ }^{\mathrm{TM}}$ and Typhidot $\mathrm{R}$ tests for typhoid fever diagnosis in a community clinic in urban Bangladesh is low ${ }^{10}$.
Typhoid remains a global public health problem, and quick accurate immunodiagnosis is needed. Rahmana et al. (2007) examined the performance of the 5-min TUBEX ${ }^{\circledR}$ O9-antibody detection kit in 243 outpatients (Mostly children and infants) in their first week of fever and 57 healthy subjects in the Bangladesh community. Based on culture results, TUBEX ${ }^{\circledR}$ was $91.2 \%(31 / 34)$ sensitive and $82.3 \%(172 / 209)$ specific in febrile subjects ${ }^{11}$. However, specificity was better in nonfebrile healthy subjects $(89.5 \%, 51 / 57)$ or in febrile individuals who serologically had dengue fever $(90.5 \%, 57 / 63)$ suggesting that some culture-negative febrile individuals could be truly typhoidal.

Typhoid fever still continues to be a major public health problem around the world. Simple, reliable, point-of-care rapid diagnostics for typhoid fever have been a long-felt need of clinicians working in endemic countries. Such tests need to be robust and suitable for use in remote areas with limited laboratory facilities and the medical staff should not require any specific technical training. The overall utility and uptake of these tests depends on their simplicity. Such tests should have limited steps and be designed to yield a simple 'posi-tive/negative' result at preset thresholds. Ideally, the results should be available within $1 \mathrm{~h}$ of the initiation of the assay, so that they can be interpreted while the patient is in attendance. Evaluation of validity of ICT for antibody in diagnosis of typhoid fever will help to overcome the gap in currently available diagnostic methods. It will also provide easy and early diagnosis of typhoid fever in resource limited country like ours.

\section{MATERIALS AND METHODS}

Laboratory based descriptive cross sectional study was done during the period of July 2012 and June 2013 in Chittagong Medical College Hospital, Department of Pediatrics \& Medicine. Chittagong Medical College Hospital, Department of Pediatrics \& Medicine. 150 clinically suspected typhoid fever patients were included in this study. Patients were allocated a study ID number at the time of enrolment. Parents of enrolled patients were asked to give informed consent and answer a brief questionnaire about clinical signs and symptoms, antimicrobial treatment, and history of typhoid fever and vaccination. On admission, a blood culture sample was taken before receiving antibiotics. Suspected colonies were identified by serological test. Antimicrobial sensitivity was assessed by the disc diffusion methods or E-test on a Muller-Hilton agar plate according to CLSI guidelines. ICT was done from day 5 onwards of appearance of fever by using SD BIOLINE Salmonella Typhi IgG / IgM Rapid test strip. The strip is designed to simultaneously detect \& differentiate IgG \& IgM antibodies to Salmonella typhi in human serum, plasma on whole blood.

\section{Interpretation of the test}

Negative: The control line is only visible on the test strip. No IgG and IgM antibodies were detected. Probably not typhoid. 
IgG Positive: The control and $\operatorname{IgG}$ line $(\mathrm{G})$ are visible on the strip. This is positive for IgG antibodies. This is indicative of previous Salmonella typhi infection (In which case current fever may not due to typhoid) or relapse or reinfection.

IgM Positive: The control line and IgM line are visible on the strip. This is positive for IgM antibodies to salmonella typhi. This is indicative of acute typhoid fever.

IgG and IgM Positive: The control line, IgM line (M) and IgG line $(G)$ are visible on the strip. This is positive for both IgM and IgG antibodies. This is indicative of acute typhoid fever.

Invalid: The control line fails to appear. Insufficient specimen volume or incorrect procedural techniques are the most likely reasons for control line failure. Repeat the test using a new test device.

\section{RESULTS}

Table 1: Categorization of the study patients according to blood culture positivity for Salmonella Typhi.

\begin{tabular}{lcc} 
Blood C/S & Number of patients & Percentage \\
Blood C/S for & 16 & 10.7 \\
Salmonella Typhoid & 134 & 89.3 \\
Negative & & \\
\hline
\end{tabular}

Table 2 : Antibiotic Sensitivity among culture positive patients $(\mathrm{n}=16)$.

\begin{tabular}{lcc} 
Sensitivity & Number of patients & Percentage \\
Ciprofloxacin (Intermediate sensitivity) & 04 & 25.0 \\
Azithromycin & 16 & 100.0 \\
Cefixime & 11 & 68.8 \\
Nalidixic acid (Intermediate sensitivity) & 09 & 56.3 \\
Ampicillin (Intermediate sensitivity) & 03 & 18.8 \\
Chloramphenicol & 06 & 37.5 \\
Ceftriaxone & 16 & 100.0 \\
Imipenam & 16 & 100.0 \\
\hline
\end{tabular}

Table 3 : ICT for typhoid fever $(n=150)$.

\begin{tabular}{lcc} 
ICT for typhoid fever & Number of patients & Percentage \\
Negative & 113 & 75.3 \\
Positive & 37 & 24.7 \\
$*$ IgM & 18 & 12.0 \\
$*$ IgM+IgG & 8 & 5.3 \\
$* * \operatorname{IgG}$ & 11 & 7.3 \\
\hline
\end{tabular}

* Indicates acute Infection

**Indicates past Infection

Table 4 : Pre Admission Antibiotic in culture positive patients ( $n=16)$.

\begin{tabular}{lcr} 
Antibiotic in culture positive & Number of patients & Percentage \\
Received & 07 & 43.8 \\
Not received & 09 & 56.2 \\
\hline
\end{tabular}

Table 5 : Comparison between ICT with blood culture for typhoid fever $(\mathrm{n}=150)$.

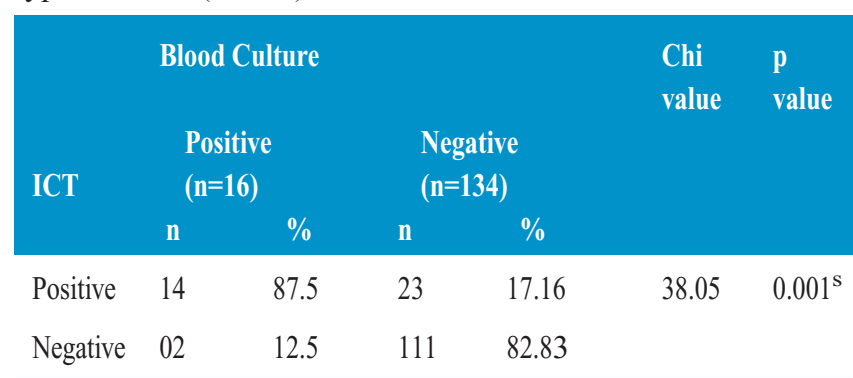

$\mathrm{S}=$ significant, $\mathrm{p}$ value reached from chi square test

Table 6 : Sensitivity, specificity, accuracy, positive and negative predictive values of ICT evaluation for prediction of typhoid fever $(\mathrm{n}=150)$.

\begin{tabular}{ll} 
Validity test & Percentage \\
Sensitivity & $88(87.5)$ \\
Specificity & $83(82.8$ \\
Accuracy & $83(83.3)$ \\
Positive Predictive Value (PPV) & $38(37.8)$ \\
Negative Predictive Value (NPV) & $98(98.2)$ \\
\hline TP=True Positive & FN=False Negative \\
TN=True Negative & FP=False Positive \\
PPV=Positive Predictive Value & \\
NPV= Positive Predictive Value & \\
DISCUSSION &
\end{tabular}

In this present study it was observed that blood $\mathrm{C} / \mathrm{S}$ for Salmonella typhi was found in $10.7 \%$ cases. A study in a superspeciality children hospital at New Delhi done by Manchanda et al. (2006) showed that a total of 56 S. typhi and $5 \mathrm{~S}$. paratyphi isolates were obtained from 673 blood culture cases that comprised $8.3 \%$ of culture positivity which is comparable with the current study ${ }^{12}$. Krishnan et al. (2009) found in their study that $70 \%$ and $30 \%$ of the isolates were Salmonella enterica serovar typhi and Paratyphi A, respectively ${ }^{13}$. Abdoel et al. (2007) found 42.5\% culturepositive patients in their study ${ }^{14}$. The final diagnosis of typhoid was based on a positive blood culture in 118 (65.9\%) patients and on clinical symptoms and signs consistent with typhoid or paratyphoid fever in 61 (34.1\%) patients found by Hatta et al. (2002). Prior antibiotic intake might have influenced yield of culture positive patients in this current study ${ }^{15}$.

In this current series it was observed that Azithromycin, Ceftriaxone and Imipenam were $100 \%$ sensitive followed by Cefixime $68.8 \%$, Nalidixic acid (Intermediately sensitive) $56.3 \%$, Chloramphenicol $37.5 \%$, Ciprofolxacin (Intermediately sensitive) $25.0 \%$ and Ampicillin (Intermediately sensitive) $18.8 \%$. Krishnan et al. (2009) found that Salmonella enterica serovar 
typhi and paratyphi A, were highly sensitive to choloramphenicol (86.0\%) ampicillin (84\%) and cotrimoxazole (88\%). Highest sensitivity was seen for cephalosporins, followed by quinolones ${ }^{13}$. In another study, Mathura et al. (2005) showed sensitivity to ceftriaxone was $100 \%$. There have been some reports of the re-emergence of the sensitivity of S. typhi to chloramphenicol and other first line drugs ${ }^{16}$. Bhatia and others reported that highest $(96 \%)$ sensitivity of Salmonella typhi to chloramphenicol in India (Bhatia, Mathur and Arora 2007) ${ }^{17}$. Another study by Yashavanth and Vidyalakshmi found that the re- emergence of chloramphenicol $(97.4 \%)$ sensitivity among the strain of S. typhi pathogens in Mangalore in 2007 (Yashavanth and Vidyalakshmi 2010) ${ }^{18}$. The above findings are comparable with the current study indicating more sensitivity of newer antimicrobial drugs.

In this study it was observed that almost a half (47.3\%) of the patients received antibiotic during pre admission period and more than a half $(52.7 \%)$ of the patients didn't received any antibiotic before hospital admission.

In this series it was observed in culture positive patients that $7(43.8 \%)$ received antibiotic and $09(56.2 \%)$ didn't receive any antibiotic during pre admission period.

In this present series it was observed that negative ICT for typhoid fever was found in $75.3 \%$ cases and positive ICT for typhoid fever was found in $24.7 \%$ cases, IgM (Acute Infection) was $12.0 \%$, IgM+IgG (Acute Infection) were $5.3 \%$ and $\operatorname{IgG}$ (Past Infection) was 7.3\%. Kawano et al. (2007) found Typhidot-IgM and $\operatorname{IgG}$ in $54.7 \%$, and $70.7 \%$ cases respectively ${ }^{19}$. Sultana (2012) has done a study in Mymensingh Medical College, Mymensingh between July, 2010 and June, 2011 including 200 individuals of different age and sex. Of them, 150 were clinically suspected cases of typhoid fever and ICT positivity was found as $71.9 \%$ having IgM antibody, $21.05 \%$ having both IgM and IgG antibody and only $7.01 \%$ having IgG antibody, which closely resembles the current study $^{20}$.

In this current study it was observed that positive ICT was found in 37 cases, out of which 14 (True positive) were blood culture positive and 23 (False positive) were blood culture negative. On the other hand a total of 113 cases with negative ICT was found, out of which 2 (False negative) were blood culture positive and 111 (True negative) were blood culture negative. The difference was statistically significant $(p<0.05)$ between two groups.

In this present study it was observed that the validity of Immunochromatographic Test (ICT) for typhoid fever having sensitivity $87.5 \%$, specificity $82.8 \%$, accuracy $83.3 \%$, positive predictive value $37.8 \%$ and negative predictive value $98.2 \%$.
ICT has been studied in many countries and they found significantly higher sensitivity and specificity (Jesudason, Esther and Mathai 2002; Pastoor et al. 2008; Anusha, Ganesh and Lalitha 2011) 9,21,22. An evaluation of ICT (Typhidot) in India was found to be $100 \%$ sensitive and $80 \%$ specific compared to blood culture as gold standard (Jesudason, Esther and Mathai 2002) ${ }^{22}$. Considering the 43 blood cultureconfirmed cases of typhoid fever as typhoid positive and the 24 other confirmed bacteremia cases as typhoid negative, ICT (Typhidot) was $67 \%$ sensitive and $54 \%$ specific, with $85 \%$ positive and $81 \%$ NPVs. Gopalakrishnan et al. (2002) evaluated the Typhidot kit and the sensitivity and specificity were found to be $82.0 \%$ and $78.0 \%$ respectively. It had a PPV and NPV of $57.7 \%$ and $90.1 \%$ with an efficiency of the test to be $72.9 \%{ }^{23}$.

\section{CONCLUSION}

The immunochromatographic Test (ICT) has been compared with the blood culture known as gold standard for diagnosis of typhoid fever. Blood culture has less sensitivity, requires wellequipped laboratory with highly skilled personnel, adequate amount of blood and at least three days for the interpretation of result \& where prior intake of antibiotic affects the result. The studied ICT, on the contrary has considerable sensitivity, is feasible at bed side by any trained health personnel and requires much less amount of blood \& time for interpreting results. Considering these points, ICT is likely to be more convenient to be applied in the remote areas deprived of satisfactory medical lab facilities.

\section{DISCLOSURE}

All the authors declared no competing interest. 


\section{REFERENCES}

1. $\quad$ Parry C.M, Hien T.T, Dougan G, White N.J, Farrar J.J. Typhoid fever. N Engl J Med. 2002;347:1770-1780.

2. Ivanoff B. Typhoid fever: Global situation and WHO recommendations. Southeast Asian J Trop Med Public Health. 1995: 26(2) :1-6.

3. World Health Organization (2003). Background document: the diagnosis, treatment and prevention of typhoid fever. WHO/V\&B/03.07. WHO, Geneva, Switzerland.

4. Gilman R.H, Terminel M, Levine M.M, Hernandez-Mendoza P, Hornick R.B. Relative efficacy of blood, urine, rectal swab, bone marrow, and rose-spot cultures for recovery of Salmonella Typhi in typhoid fever. Lancet.1975;1(7918):1211-1213.

5. Guerra-Caceres J.G, Gotuzzo-Herencia E, Crosby-Dagninio E, Miro-Quesada M, Carrillo-Parodi C. Diagnostic value of Expert Rev. Anti Infect. Ther. 2011;9(6) : 722 .

6. Choo K.E, Oppenheimer S.J, Ismail A.B, Ong K.H. Rapid serodiagnosis of typhoid fever by dot enzyme immunoassay in an endemic area Clin. Infect. Dis.1994;19:172-176.

7. Jackson A.A, Ismail A, Ibrahim T.A, Kader Z.S, Nawi, N.M. Retrospective review of dot enzyme immunoassay test for typhoid fever in an endemic area. Southeast Asian J Trop Med Public Health.1995; 26(4): 625-630.

8. Narayanappa D, Sripathi R, Jagdishkumar K and Rajani H.S. Comparative Study of Dot Enzyme Immunoassay (Typhidot-M) and Widal Test in the Diagnosis of Typhoid Fever. Indian Pediatrics. 2010; 47: 17.

9. Pastoor R, Hatta M, Abdoel TH, Smits HL. A simple, rapid, and affordable point-of care test for the serodiagnosis of typhoid fever. Diagnostic Microbiology and Infectious Disease.2008;61(2):129-134.

10. Naheed A, Ram P.K, Brooks W.A, Mintz E.D, Hossain A, Parsons M.M, Luby S.T, Breiman R.F et al. Clinical value of Tubex ${ }^{\mathrm{TM}}$ and

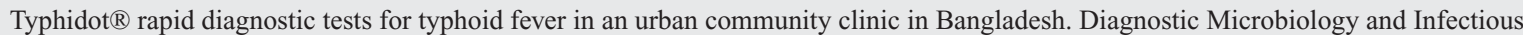
Disease. 2008; 61: 381-386.

11. Rahmana M, Siddiqueb A.K, Tamc F.C, Sharmina S, Rashida H, Iqbalb A, Ahmedb S, Naira, G.B, Chaignatd C, Limc P et al. Rapid detection of early typhoid fever in endemic community children by the TUBEX ${ }^{\circledR}$ O9-antibody test. Diagnostic Microbiology and Infectious Disease 2007; $58: 275-281$

12. Manchanda V, Bhalla P, Sethi M, Sharma VK. Treatment of enteric fever in children on the basis of current trends of antimicrobial susceptibility of Salmonella enterica serovar typhi and paratyphi A. Indian J Med Microbiol. 2006; 24(2): 101-106.

13. Krishnan P, Stalin M, Balasubramanian. S. Changing trends in antimicrobial resistance of Salmonella enterica serovar typhi and salmonella enterica serover parathyphi A in Chennai. Indian J Pathol Microbiol. 2009:1.

14. Abdoel T.H, Pastoor R, Smits H.L, Hatta M. Laboratory evaluation of a simple and rapid latex agglutination assay for the serodiagnosis of typhoid fever. Transactions of the Royal Society of Tropical Medicine and Hygiene. 2007;101:1032-1038.

15. Hatta M, Goris M.G.A, Heerkens E, Goosken, J and Smits H.L. Simple Dipstick Assay For The Detection Of Salmonella Typhi-Specific Igm Antibodies And The Evolution of The Immune Response In Patients With Typhoid Fever. Am. J. Trop. Med. Hyg. 2002;66(4):416-421.

16. Mathura K.C, Chaudhary D, Simkhada R, Pradhan M, Shrestha P, Gurubacharya D.L. Study of clinical profile and antibiotic sensitivity pattern in culture positive typhoid fever cases. Kathmandu Univ Med J (KUMJ). 2005;1.

17. Bhatia JK, Mathur AD, Arora AD. Re-emergence of Chloramphenicol sensitivity in enteric fever. 2007;63(3): 212-214.

18. Yashavanth R. and Vidyalakshmi K. The Re-Emergence Of Chloramphenicol Sensitivity Among Enteric Fever Pathogens In Mangalore, Journal of Clinical and Diagnostic Research. 2010;4:3106-3108.

19. Kawano R.L, Leano, S.A and Agdamag D.M.A.. Comparison of Serological Test Kits for Diagnosis of Typhoid Fever in the Philippines. Journal of Clinical Microbiology. 2007; 246-247.

20. Sultana S. Comparison of different test methods including polymerase chain reaction for early and reliable diagnosis of typhoid fever. Thesis part of M Phil. Department of Microbiology, Mymensingh Medical College, Mymensingh, Bangladesh. 2012.

21. Anusha R, Ganesh R and Lalitha J. Comparison of a rapid commercial test, Enterocheck WB, with automated blood culture for diagnosis of typhoid fever. Annals of Tropical Paediatrics: International Child Health. 2011;31(3) :231-234

22. Jesudason M, Esther E, Mathai E. Typhi dot test to detect IgG \& IgM antibodies in typhoid fever. Indian J Med Res. 2002; 116:70-72.

23. Gopalakrishnan V, Sekhar W.Y, Soo E.H, Vinsent R.A, Devi S. Typhoid Fever in Kuala Lumpur and a Comparative Evaluation of Two Commercial Diagnostic Kits for the Detection of Antibodies to Salmonella typhi. Singapore Med J. 2002; 43(7) :354-358. 